\title{
Inhibition and disinhibition of return: Evidence from temporal order judgments
}

\author{
BRADLEY S. GIBSON and HOWARD EGETH \\ Johns Hopkins University, Baltimore, Maryland
}

\begin{abstract}
In the present study the temporal order judgment (TOJ) task was used to investigate whether or not inhibition of return (IOR) affects perceptual processing. Previous failures to obtain IOR in the TOJ task have been taken to suggest that IOR does not affect perceptual processing (e.g., Maylor, 1985). The present study showed that IOR is modulated by the temporal disparity between successive targets as well as the relative order in which they appear at cued and uncued locations. Consequently, IOR affects TOJs in some conditions but not in others. The selective occurrence of IOR in the TOJ task provides converging support for the notion that IOR does affect perceptual processing, and also accounts for the previous failures to observe IOR in the TOJ task. Moreover, these and other results suggest that inhibitory processing at the cued location can be disinhibited when stimulation occurs at other locations.
\end{abstract}

Visual information processing often involves an interplay between facilitatory and inhibitory processes. One such interaction was recently revealed in a simple cuetarget paradigm. Posner and Cohen (1984) showed that a cue consisting of a transient luminance change displayed in the periphery produced a dual effect on the subsequent detection of luminance targets presented there. One effect, which dominated at short cue lead times, was characterized by detection latencies that were faster when the target appeared at the cued location than when it appeared elsewhere. The other effect, which dominated at longer cue lead times, showed the opposite pattern; that is, detection latencies were slower when the target appeared at the cued location than when it appeared elsewhere. This biphasic pattern of detection latencies has been taken to signify the joint operation of two independent processes, one facilitatory and the other inhibitory.

According to Posner and Cohen (1984; see also Eriksen \& Yeh, 1985; Jonides, 1981), attention was initially summoned to the target's location by the peripheral cue, thus accounting for the facilitation observed at short cue lead times. Likewise, the inhibitory component observed at longer cue lead times was also thought to be invoked by an aspect of the cue, but this component was argued to operate via some mechanism other than attentional orienting (Posner \& Cohen, 1984; but see Maylor, 1985;

\footnotetext{
Support was provided by grants from the National Institute of Mental Health (T32-MH18215) to Johns Hopkins University, and from the National Science Foundation (BNS-8919554) and the Air Force Office of Scientific Research (ISSA-92-0041) to the second author. The authors would like to thank Laura Carlson-Radvansky, Kyle Cave, Lester Krueger, Tram Neill, and Kimron Shapiro for helpful comments on an earlier version of this article. Correspondence should be addressed to Bradley S. Gibson, Department of Psychology, University of Notre Dame, Notre Dame, IN 46556 (e-mail: gibson@jhuvms.hcf.jhu.edu).
}

Maylor \& Hockey, 1985). Considerable debate has erupted concerning the nature of the inhibitory component, which has come to be known as inhibition of return (IOR). In fact, recent evidence suggests that IOR may not be a unitary phenomenon, but may instead reflect distinct forms of inhibition that affect different processing systems. In particular, cuing may inhibit both eye movements (Posner, Rafal, Choate, \& Vaughan, 1985; Rafal, Calabresi, Brennan, \& Sciolto, 1989) and stimulus detection (Posner \& Cohen, 1984) following long cue lead times.

The duality of IOR has recently been demonstrated by Abrams and Dobkin (1994; see also Rafal, Egly, \& Rhodes, 1994). In Abrams and Dobkin's study, subjects made speeded eye movements to one of two peripheral locations. On each trial, one location was exogenously cued. After a $960-\mathrm{msec}$ interval, an imperative stimulus appeared, indicating the location to which a saccade was to be made. On some trials, the imperative stimulus was an arrow, presented at fixation, that pointed equiprobably to either the cued or the uncued location; on the remaining trials, the imperative stimulus was a peripheral luminance increment presented equiprobably at either the cued or the uncued location. Thus, only the latter condition required that a peripheral stimulus be detected at the cued or the uncued location before the correct oculomotor response could be elicited.

Abrams and Dobkin (1994) found that IOR [cued response time (RT) - uncued RT] was approximately three times as large when the imperative stimulus was a peripheral luminance increment as when it was a central arrow. These results suggest that approximately two thirds of the IOR observed when the imperative stimulus was a peripheral luminance increment was due to an impairment in detecting a stimulus displayed at the cued location, with the remaining third due to an impairment in making a saccade to the cued location. 
Despite this evidence, however, the conclusion that IOR affects perceptual processing remains controversial. One reason for this controversy involves studies of temporal order (Kwak, 1992; Maylor, 1985; Posner et al., 1985). The temporal order judgment (TOJ) task is similar to the simple detection task originally used by Posner and Cohen (1984), except that, following the cue, two successive targets ( $\mathrm{T} 1$ and $\mathrm{T} 2$ ) are displayed at separate locations on each trial. The task of subjects is to determine which target appears first, and accuracy is typically emphasized over speed. TOJs provide an important source of converging evidence for facilitatory and inhibitory processes, because such judgments are affected by the temporal characteristics of visual information processing.

For instance, consider the two conditions illustrated in Figure 1. Each display contains a central fixation cross and two peripheral squares. On each trial, one square is exogenously cued (brightened). Cue lead time refers to the interval between onset of the cue and onset of $\mathrm{T} 1$; target asynchrony refers to the interval between onset of $\mathrm{T} 1$ and onset of T2. Note that in this illustration, as in the experiments to follow, T1 remains on the screen along with T2 until the subject responds. In the firstcued condition, $\mathrm{T} 1$ is displayed at the cued location and $\mathrm{T} 2$ is displayed at the uncued location. In the secondcued condition, $\mathrm{T} 1$ is displayed at the uncued location and $\mathrm{T} 2$ is displayed at the cued location.

At short cue lead times, cuing is expected to facilitate processing at the cued location. TOJs should therefore be easier in the first-cued condition than in the secondcued condition because the perceived temporal interval between $\mathrm{T} 1$ and $\mathrm{T} 2$ would be expanded (by facilitatory processing of $\mathrm{T} 1$ ) in the former condition and compressed (by facilitatory processing of T2) in the latter condition. At long cue lead times, cuing is expected to inhibit processing at the cued location. TOJs should therefore be harder in the first-cued condition than in the second-cued condition, because the perceived temporal interval between $\mathrm{T} 1$ and $\mathrm{T} 2$ would be compressed (by inhibitory processing of $\mathrm{T} 1$ ) in the former condition and expanded (by inhibitory processing of $\mathrm{T} 2$ ) in the latter condition.

However, while recent studies of temporal order have suggested that visual information processing is facilitated following short cue lead times (Hikosaka, Miyauchi, \& Shimojo, 1993; Maylor, 1985; Stelmach \& Herdman, 1991), they have failed to show that visual information processing is inhibited following long cue lead times (Kwak, 1992; Maylor, 1985; Posner et al., 1985). The failure to find an inhibitory component in the TOJ task at long cue lead times (Kwak, 1992; Maylor, 1985; Posner et al., 1985) therefore fails to provide converging support for the notion that IOR impairs perceptual processing.

Note, however, that several differences exist between the TOJ task and the simple detection task which may affect the manifestation of IOR. First, two successive targets are displayed on each trial in the TOJ task, whereas only a single target is displayed on each trial in the simple detection task. Second, accuracy has served as the primary measure of IOR in the TOJ task (Kwak, 1992; Maylor, 1985; Posner et al., 1985), whereas latency has served as the primary measure of IOR in the simple detection task (Maylor, 1985; Maylor \& Hockey, 1985; Posner \& Cohen, 1984; Rafal et al., 1989). In the present article, we attempt to clarify the nature of IOR within the context of the TOJ task by determining whether IOR is affected by the appearance of multiple target stimuli as well as whether IOR is dependent on how performance is measured.
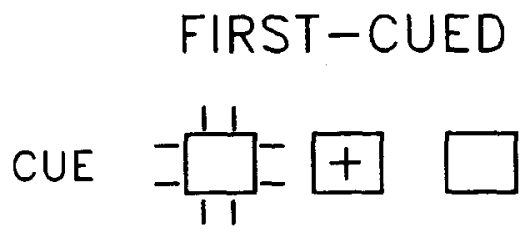

T1
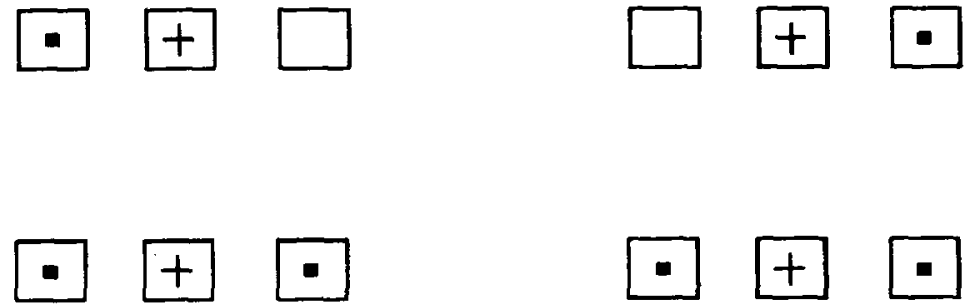

T2

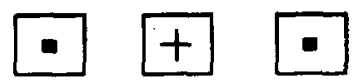

Figure 1. The two basic types of trials used in the temporal order judgment task. Note that cue lead time refers to the interval between onset of the cue and onset of $\mathrm{T} 1$; target asynchmny refers to the interval between onset of $\mathrm{T} 1$ and onset of $\mathrm{T} 2$. 


\section{EXPERIMENT 1}

The purpose of Experiment 1 was twofold. First, we manipulated the number of target stimuli displayed following a peripheral cue to determine whether IOR would be obtained only when a single target was presented. On half of the trials, a single target (T1) appeared randomly at either the cued or the uncued location following a variable cue lead time. The remaining trials were identical to these single-target trials, except that a second target (T2) was also presented at the opposite location following a variable-target asynchrony, as shown in Figure 1. Hence, on any given trial, subjects could not predict whether one target or two would follow the cue. Regardless of the number of targets, the subjects were instructed to press a key corresponding to the location of the first target.

The second purpose of Experiment 1 was to determine whether the manifestation of IOR on double-target trials depended on how performance was measured. Thus, unlike previous TOJ studies (Hikosaka et al., 1993; Kwak, 1992; Maylor, 1985; Posner et al., 1985; Stelmach \& Herdman, 1991), accuracy was not emphasized over speed in this experiment; rather, subjects were instructed to respond as quickly as possible while trying to keep errors to a minimum. This allowed the standard latency measure of IOR to be obtained on single-target trials as well as on double-target trials.

\section{Method}

Subjects. Thirteen students from Johns Hopkins University participated in this study for $\$ 3$. All subjects reported normal or corrected-to-normal vision.

Stimuli and Apparatus. Stimulus displays consisted of a central fixation "+" and two outline squares positioned to the left and right of fixation. At a viewing distance of $50 \mathrm{~cm}$, the fixation "+" subtended $0.57^{\circ}$ of visual angle and the two squares subtended $1.49^{\circ} \times 1.49^{\circ}$ of visual angle. Distance between each square and fixation was $4.69^{\circ}$. Cuing consisted of brightening one peripheral square by increasing the thickness of the lines making up the square from $0.11^{\circ}$ to $0.23^{\circ}$. The targets were small squares $\left(0.23^{\circ}\right.$ $\times 0.23^{\circ}$ ) that appeared in the center of each peripheral square. Displays were presented on a standard VGA monitor (white on black).

Procedure. Each trial began with the presentation of the fixation "+" and the two squares for 500 msec. The subjects were instructed to keep their eyes fixed on the "+" throughout each trial, although eye movements were not monitored. Following this initial display, one of the two squares was brightened for $100 \mathrm{msec}$. Following a randomly determined cue lead time of 100,300 , or $700 \mathrm{msec}, \mathrm{T} 1$ appeared randomly at either the cued or uncued location. On single-target trials, T1 was the only target to appear; however, on double-target trials, T2 was displayed in the opposite square following a randomly determined target asynchrony of 14 , 43 , or 114 msec. Both T1 and T2 (if it was presented) remained on the screen until the subjects responded. This type of stimulus presentation eliminated any apparent motion that might otherwise have occurred had $\mathrm{T} 1$ disappeared before $\mathrm{T} 2$ was displayed. The subjects were instructed to press the left shift key if the first target appeared in the left square and the right shift key if the first target appeared in the right square.

The subjects served in a single experimental session consisting of five blocks of 72 trials. The first block served as practice and was not included in the results. Within each block of trials, an equal number of single-target and double-target trials was shown at each of the three cue lead times. For each cue lead time, an equal number of double-target trials was shown at each of the three target asynchronies.

\section{Results}

Single-target trials. We began by establishing the typical pattern of facilitation and inhibition observed when a single target follows the cue (Maylor, 1985; Maylor \& Hockey, 1985; Posner \& Cohen, 1984). Mean correct RT and percent errors on single-target trials can be seen in Table 1 listed as a function of cue lead time and cuing. Mean correct RT and percent errors were each submitted to a $3 \times 2$ repeated measures analysis of variance (ANOVA) with cue lead time and cuing as within-subjects factors. Errors were uniformly low on single-target trials, ranging from $0.32 \%$ to $1.61 \%$; there were no significant effects (all $p s>.20$ ).

The ANOVA performed on mean correct RT revealed a significant main effect of cue lead time $[F(2,24)=$ $44.27, p<.001]$, indicating that RT decreased as cue lead time increased. This pattern of RTs is typically attributed to a general alerting effect induced by the cue (see, e.g., Posner \& Boies, 1971). As expected, there was also a significant cue lead time $\times$ cuing interaction $[F(2,24)=5.74, p<.01]$. Latencies were faster in the cued condition than in the uncued condition when cue lead time was $100 \mathrm{msec}[F(1,12)=4.86, p<.05]$. However, RTs were slower in the cued condition than in the uncued condition when cue lead time was $700 \mathrm{msec}$ $[F(1,12)=4.57, p=.05]$. There was no difference observed at the intermediate $(300-\mathrm{msec})$ cue lead time $(F<1)$. Hence, the typical pattern of early facilitation and late inhibition initially reported by Posner and Cohen (1984; Maylor, 1985; Maylor \& Hockey, 1985) was successfully observed in the present experiment when a single target was displayed.

Double-target trials. Mean correct RT and percent errors on double-target trials are depicted in Figure 2 as a function of target asynchrony, cue lead time, and cuing. Both mean correct RT and percent errors were submitted to a $3 \times 3 \times 2$ repeated measures ANOVA, with target asynchrony, cue lead time, and cuing as within-subjects factors.

Errors. As expected, fewer errors were committed in the first-cued condition than in the second-cued condition $[F(1,12)=72.91, p<.0001]$. The main effect of

Table 1

Mean Correct Response Time and Percent Errors as a Function of Cue Lead Time and Cuing on Single-Target Trials in Experiment 1

\begin{tabular}{lccc}
\hline & \multicolumn{3}{c}{ Cue Lead Time (in Milliseconds) } \\
\cline { 2 - 4 } Cuing & 100 & 300 & 700 \\
\hline Cued & & & \\
RT & 489 & 441 & 431 \\
$\quad$ Errors (\%) & 0.32 & 0.65 & 0.96 \\
Uncued & & & \\
RT & 516 & 450 & 411 \\
Errors (\%) & 0.97 & 0.65 & 1.61 \\
\hline
\end{tabular}


cuing was qualified by a target asynchrony $x$ cuing interaction $[F(2,24)=11.10, p<.0001]$, a cue lead time $\times$ cuing interaction $[F(2,24)=43.66, p<.0001]$, and a target asynchrony $\times$ cue lead time $\times$ cuing interaction $[F(4,48)=2.70, p<.05]$.

As can be seen in Figure 2 at the shortest $(100-\mathrm{msec})$ cue lead time, for each target asynchrony, substantially fewer errors were committed in the first-cued condition than in the second-cued condition, although the magnitude of this difference decreased as target asynchrony increased, perhaps because errors were at floor in the 114-msec target asynchrony condition. This finding replicates several earlier studies (Hikosaka et al., 1993; Maylor, 1985; Stelmach \& Herdman, 1991), and suggests that attention speeds visual information processing at the cued location. Importantly, however, there was no evidence that TOJs were influenced by IOR following long cue lead times. Although the difference between the first-cued condition and the second-cued condition decreased across cue lead time at each of the three target asynchronies, performance was never impaired in the first-cued condition relative to the second-cued condition. This finding replicates earlier findings as well (Kwak, 1992; Maylor, 1985; Posner et al., 1985), and thus casts doubt on the perceptual nature of IOR.

Mean correct RT. Because latency has served as the primary measure of IOR in previous studies (e.g., Abrams \& Dobkin, 1994; Maylor, 1985; Maylor \&
Hockey, 1985; Posner \& Cohen, 1984; Rafal et al., 1989), it was of interest to determine whether RTs followed the same pattern as errors on double-target trials. ${ }^{1}$ There were significant main effects of target asynchrony $[F(2,24)=29.17, p<.0001]$, cue lead time $[F(2,24)=$ $11.49, p<.0005]$, and cuing $[F(1,12)=26.14, p<$ $.0005]$. In addition, there was also a significant target asynchrony $\times$ cue lead time interaction $[F(4,48)=$ $2.52, p=.05]$ as well as a significant cue lead time $\times$ cuing interaction $[F(2,24)=4.07, p<.05]$. The target asynchrony $\times$ cue lead time $\times$ cuing interaction, however, was not significant $(F<1)$.

The cue lead time $\times$ cuing interaction reflected faster RTs in the first-cued condition than in the second-cued condition at both shorter cue lead times $(p<.0005$ for 100 -msec cue lead time and $p<.005$ for 300 -msec cue lead time). However, there was no difference between the first-cued and second-cued conditions when cue lead time was $700 \mathrm{msec}(p>.10)$. Thus, latency measures were found to dovetail with accuracy measures on double-target trials. Together, these results suggest that IOR does not affect TOJs.

RT comparison between single-target and doubletarget trials. We next compared latencies across the two types of trials. Because target asynchrony did not interact with cue lead time and cuing on double-target trials, we averaged RT across target asynchrony and performed a repeated measures ANOVA with target type (single vs.

\section{Target Asynchrony}

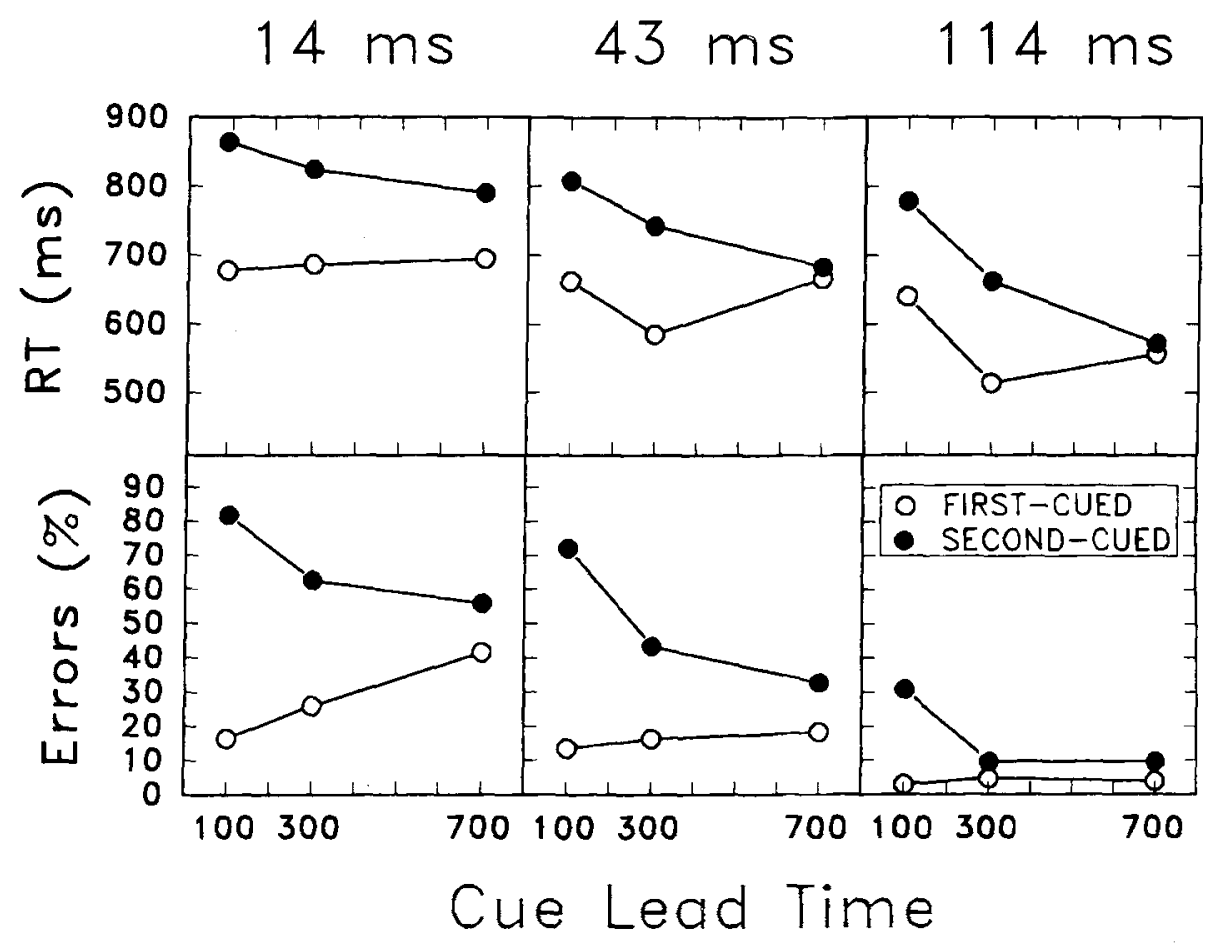

Figure 2. Mean correct response time and percent errors plotted as a function of target asynchrony, cue lead time, and cuing on double-target trials in Experiment 1. 
double targets), cue lead time, and cuing as the three repeated factors.

As might be expected, turning a simple detection task into a TOJ task by displaying a second target at the opposite location made responses substantially slower, as indicated by a main effect of target type $[F(1,12)=59.03$, $p<.0001]$. There was also a significant cue lead time $\times$ cuing interaction $[F(2,24)=6.35, p<.01]$ and a target type $\times$ cuing interaction $[F(1,12)=21.70, p<.001]$, indicating a greater cuing effect on double-target trials than on single-target trials.

\section{Discussion}

The results obtained on single-target trials suggest that IOR was present but did not appear to affect (in the expected way) the processing of targets displayed at the cued location on double-target trials. On double-target trials, subjects were both faster and more accurate in the first-cued condition than in the second-cued condition at cue lead times of 100 and $300 \mathrm{msec}$, but performance was not impaired in the first-cued condition when cue lead time was $700 \mathrm{msec}$. However, this need not imply that IOR was completely eliminated on double-target trials. It would, indeed, be puzzling if it were completely eliminated, given that the same cues gave rise to IOR (as measured by latency) on single-target trials. Nevertheless, neither the standard latency measure nor one based on errors provided evidence for IOR on doubletarget trials in the present experiment, despite the fact that those trials were randomly intermixed with singletarget trials.

Apparently, the appearance of two targets, instead of one, altered IOR. Note, however, that the expected interaction of cue lead time and cuing was obtained on double-target trials; the difference between our data and previous (single-target) data that have been taken to indicate IOR is the failure of our first-cued and secondcued functions to cross at long cue lead times.

One reason the first-cued and second-cued functions did not cross at the long cue lead time may be that IOR is dependent on the relative order in which target stimuli are displayed at cued and uncued locations. More specifically, IOR may have occurred in the first-cued condition but not in the second-cued condition (perhaps because the process generating IOR has a very short "memory" and operates on only the most recently stimulated location; see, e.g., Pratt \& Abrams, 1993). If IOR occurred in the first-cued condition, but not in the secondcued condition, then the failure of the curves to cross suggests that something may have impaired processing in the second-cued conditions at the long cue lead time.

The impairment in the second-cued condition may have occurred because the presumed elimination of IOR in this condition allowed any remaining facilitatory processes to operate on $\mathrm{T} 2$ at the cued location. As such, this account assumes that facilitation normally persists at long cue lead times (see, e.g., Egly, Rafal, \& Henik, 1992) but is typically masked by more dominant inhibitory processes. Thus, when those more dominant in- hibitory processes are released, facilitation may compress the temporal interval between the two target stimuli in the second-cued condition at long cue lead times as well as at short cue lead times. In short, the performance impairment expected in the first-cued condition (due to inhibitory processing of $\mathrm{T} 1$ ) may have been offset by impaired performance in the second-cued condition (due to facilitatory processing of T2). This hypothesis was investigated in the next experiment.

\section{EXPERIMENT 2}

The hypothesis that IOR occurred in the first-cued condition but not in the second-cued condition was tested by including a neutral baseline condition in Experiment 2 . These trials were identical to the previous doubletarget trials, with the exception that neither peripheral square was cued. Rather, a central square, drawn around fixation, was cued in the neutral condition.

Using a similar neutral condition in conjunction with a simple detection task, Rafal et al. (1989) showed that cued RTs were significantly slower than neutral RTs following long cue lead times. Hence, if the failure to observe IOR on double-target trials in Experiment 1 was due to impaired performance in the second-cued condition, then both the first-cued and second-cued conditions should be impaired relative to the neutral condition following long cue lead times in the present experiment.

\section{Method}

Subjects. Eleven students from Johns Hopkins University participated in this study for $\$ 5$. All subjects reported normal or corrected-to-normal vision.

Stimuli and Apparatus. The stimulus displays were identical to those used in Experiment 1, with the exception that a third outline square was drawn around the central fixation "+."

Procedure. The procedure was identical to that of Experiment 1, with the following exceptions: (1) Only double-target trials were shown in Experiment 2. (2) On half of the trials, one of the two peripheral squares was brightened for $100 \mathrm{msec}$; on the other half, the central square was brightened for $100 \mathrm{msec}$. (3) Cue lead times of $100,200,300,400,500,600$, and $700 \mathrm{msec}$ were used to allow a more detailed analysis of the effects of cuing over time. However, target asynchrony was held constant at $114 \mathrm{msec}$ in order to accommodate the increased number of cuing conditions and cue lead times.

The subjects served in a single experimental session consisting of 10 blocks of 56 trials. The first block served as practice and was not included in the results.

\section{Results}

The main purpose of this experiment was to determine whether IOR could selectively occur in just the first-cued condition. Mean correct RT and percent errors in each of the first-cued, second-cued, and neutral conditions are depicted in Figure 3 as a function of cue lead time.

Errors. There were main effects of cue lead time and cuing $[F(6,60)=16.83, p<.0001$ and $F(2,20)=11.45$, $p<.0005$, respectively], and there was also a signficant cue lead time $\times$ cuing interaction $[F(12,120)=19.00$, 
$p<.0001]$. Because our prime interest concerned the relationship of the first-cued and second-cued conditions to the neutral condition, we conducted two additional ANOVAs.

We first compared errors in the first-cued condition with errors in the neutral condition. There was a marginally significant main effect of cue lead time $[F(6,60)=$ $2.19, p<.06]$, and there was a significant cue lead time $\times$ cuing interaction $[F(6,60)=3.03, p<.02]$. An analysis of simple effects indicated that slightly more errors were committed in the neut ral condition than in the firstcued condition following a cue lead time of $100 \mathrm{msec}$ $(p<.06)$. There were no differences between the firstcued condition and the neutral condition at the remaining cue lead times (all $p \mathrm{~s}>.30$ ). Note that the marginally significant effect of cuing following a cue lead time of $100 \mathrm{msec}$ and the failure to observe an increase in errors in the first-cued condition relative to the neutral condition following long cue lead times might be due to a floor effect.

When errors in the second-cued condition were compared with errors in the neutral condition, there were significant main effects of cue lead time $[F(6,60)=25.10$, $p<.0001]$ and cuing $[F(1,10)=26.00, p<.0005]$. There was also a significant cue lead time $\times$ cuing interaction

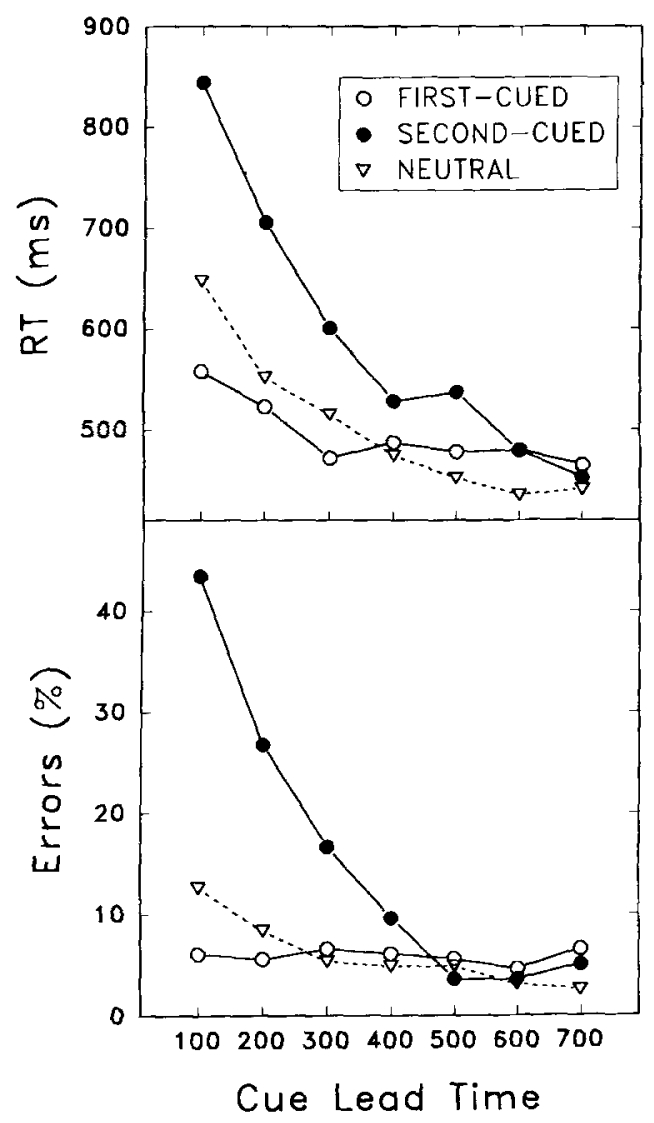

Figure 3. Mean correct response time and percent errors plotted as a function of cue lead time and cuing in Experiment 2. Target asynchrony was $114 \mathrm{msec}$.
$[F(6,60)=16.70, p<.0001]$. An analysis of simple effects revealed that more errors were committed in the second-cued condition than in the neutral condition following the four shortest cue lead times (all $p$ s $<.05$ ), but they did not differ following the three longest cue lead times (all $p s>.20$ ). Again, the failure to find differences between the second-cued condition and the neutral condition following long cue lead times might be due to a floor effect.

Mean correct RT. There were significant main effects of cue lead time $[F(6,60)=57.53, p<.0001]$ and cuing $[F(2,20)=33.59, p<.0001]$. In addition, there was also a significant cue lead time $\times$ cuing interaction $[F(12,120)=$ $13.94, p<.0001]$. Again, we compared RT in the firstcued condition with RT in the neutral condition. There was a significant main effect of cue lead time $[F(6,60)=$ $26.33, p<.0001]$, but more importantly there was also a significant cue lead time $\times$ cuing interaction $[F(6,60)=$ $10.91, p<.0001]$. As shown in Figure 3 , the typical pattern of results observed on single-target trials was obtained on double-target trials when RTs in the first-cued condition were compared with RTs in the neutral condition. That is, facilitation at short cue lead times (less than $400 \mathrm{msec}$ ) gave way to inhibition at longer cue lead times (greater than $400 \mathrm{msec}$ ). An analysis of simple effects indicated that RTs in the first-cued condition were significantly faster than RTs in the neutral condition following cue lead times of 100 and $300 \mathrm{msec}$, but significantly slower following cue lead times of 500,600 , and $700 \mathrm{msec}$ (all $p s<.05$ ). Unlike previous TOJ studies, which measured only accuracy and did not compare the firstcued condition to a neutral control condition (Maylor, 1985; Posner et al., 1985), these findings suggest that IOR does indeed affect TOJs, at least when latency is measured.

Moreover, comparison of the second-cued and baseline conditions suggests why the standard latency measure of IOR was not observed in Experiment 1. There were main effects of cue lead time $[F(6,60)=68.50, p<$ $.0001]$ and cuing $[F(1,10)=82.37, p<.0001]$. There was also a significant cue lead time $\times$ cuing interaction $[F(6,60)=8.16, p<.0001]$. Analysis of simple effects indicated that RTs in the second-cued condition were slower than RTs in the neutral condition at all cue lead times (all $p s<.02$ ) except following the cue lead time of $700 \mathrm{msec}(p>.30)$. The most interesting aspect of these findings is that RTs in the second-cued condition remained slower than RTs in the neutral condition, even at cue lead times (500 and $600 \mathrm{msec}$ ) that indicated the presence of IOR at the cued location.

\section{Discussion}

The results of Experiment 2 provided affirmative evidence that IOR does depend on the relative order in which $\mathrm{T} 1$ and $\mathrm{T} 2$ appear at cued and uncued locations. In particular, RTs in the first-cued condition were slower than RTs in the neutral condition at long cue lead times (greater than $400 \mathrm{msec}$ ), which is usually taken to indicate IOR. In contrast, IOR did not appear to affect per- 
formance in the second-cued condition: Latencies in the second-cued condition were also found to be slower than RTs in the neutral condition across all but the longest of cue lead times. Note that this latter impairment is likely due to facilitatory processing at the cued location. Impaired performance was predicted in the second-cued condition at short cue lead times because attentional facilitation has the effect of compressing the perceived temporal interval between the two targets, thereby making it more difficult to determine which target appeared first. Although this compression apparently decreased as a function of cue lead time in the second-cued condition, as indicated by decreasing impairment relative to the neutral condition, it continued to exert a significant effect on latencies even after approximately $600 \mathrm{msec} .^{2}$

Thus, the results of Experiment 2 suggest that IOR affects the processing of $\mathrm{T} 1$ at the cued location. In contrast, when $T 1$ is displayed at the uncued location, IOR does not affect the processing of $\mathrm{T} 2$ at the cued location. Rather, the presentation of $\mathrm{T} 1$ at the uncued location appears to release IOR at the cued location, leaving any remaining facilitation to affect the processing of $\mathrm{T} 2$ at the cued location.

\section{EXPERIMENT 3}

Experiment 2 showed that IOR was affected by the relative order in which target stimuli appeared at the cued and uncued locations; however, the temporal interval between $\mathrm{T} 1$ and $\mathrm{T} 2$ was of a relatively long and fixed duration in that experiment. Hence, it is important to determine whether target asynchrony also affects IOR. Such evidence would further clarify the functional nature of IOR when multiple stimuli are presented.

This issue is also relevant to the question of whether IOR can affect accuracy, the typical measure of performance in the TOJ task (Hikosaka et al., 1993; Kwak, 1992; Maylor, 1985; Posner et al., 1985; Stelmach \& Herdman, 1991). As shown in Experiment 2, latency was impaired in the first-cued condition relative to the neutral condition following long cue times; however, there was no corresponding error effect associated with this RT impairment. Of course, it is likely that error rates were at floor in Experiment 2 given the relatively long $(114 \mathrm{msec})$ target asynchrony used in this experiment; thus, any difference in accuracy between the first-cued and neutral conditions may have been obscured. Hence, meaningful accuracy data may be obtained only when shorter target asynchronies are used. However, if IOR is released in the first-cued condition at short target asynchronies, then accuracy may not reflect IOR even at short target asynchronies.

\section{Method}

Subjects. Nine students from Johns Hopkins University participated in this study either for $\$ 5$ or for course credit. All subjects reported normal or corrected-to-normal vision.

Procedure. All aspects of Experiment 3 were identical to those of Experiment 2, except that three levels of target asynchrony were used: 43,72 , and $100 \mathrm{msec}$. In addition, only long cue lead times $(500,700$, and $900 \mathrm{msec})$ were used in this experiment. This range of values included two cue lead times at which IOR had been observed in Experiment 2 (500 and $700 \mathrm{msec}$ ) and one longer value (900 msec). Moreover, as shown in Experiment 2, the 500-msec cue lead time should allow any impairment in the second-cued condition to be observed. As before, both target asynchrony and cue lead time were randomly presented within each block.

Subjects served in a single experimental session consisting of eight blocks of 72 trials. The first block served as practice and was not included in the results.

\section{Results}

Mean correct RT and percent errors are shown in Figure 4 as a function of target asynchrony, cue lead time, and cuing.

Errors. There were main effects of target asynchrony $[F(2,16)=59.68, p<.0001]$ and cue lead time $[F(2,16)=$ $4.68, p<.05]$. Simple effects tests indicated that more errors were committed in the 43-msec target asynchrony condition $(M=23.46 \%)$ than in either the $72-\mathrm{msec}$ condition $(M=9.82 \%)$ or the $100-\mathrm{msec}$ condition $(M=$ $5.55 \%)$. The latter two conditions were also significantly different from one another (all $p \mathrm{~s}<.02$ ). The main effect of cue lead time indicated that more errors were committed in the 500-msec condition $(M=15.82 \%)$ than in the 900 -msec condition $(M=10.49 \% ; p<.05)$. The 700 -msec condition $(M=12.52 \%)$ did not differ from either the 500-msec condition or the $900-\mathrm{msec}$ condition (both $p s>.08$ ).

Mean correct RT. There were significant main effects of target asynchrony $[F(2,16)=15.23, p<.0005]$ and cue lead time $[F(2,16)=17.94, p<.0002]$. In addition, there was also a significant target asynchrony $x$ cuing interaction $[F(4,32)=2.82, p<.05]$ and a significant cue lead time $\times$ cuing interaction $[F(2,16)=6.96$, $p<.0005]$. As in Experiment 2, we performed two additional ANOVAs to clarify these interactions.

In the first ANOVA, we compared RT in the first-cued condition with RT in the neutral condition at each of the three target asynchronies and cue lead times. There were significant main effects of target asynchrony $[F(2,16)=$ $11.44, p<.001]$ and cue lead time $[F(2,16)=7.94, p<$ $.005]$. More importantly, there was also a significant target asynchrony $\times$ cuing interaction $[F(2,16)=4.42, p<$ .05]. Simple effects tests indicated that the first-cued condition did not differ from the neutral condition in either the 43-msec or the 72-msec target-asynchrony conditions (both $p s>.30$ ). However, a net inhibitory effect did emerge in the first-cued condition relative to the neutral condition in the 100 -msec target-asynchrony condition $(p<.05)$. Neither the cue lead time $\times$ cuing interaction nor the target asynchrony $\times$ cue lead time $\times$ cuing interaction was significant (both $p s>.30$ ).

In the second ANOVA, we compared RT in the secondcued condition with RT in the neutral condition at each of the three target asynchronies and cue lead times. There were main effects of target asynchrony $[F(2,16)=$ $17.19, p<.0002]$ and cue lead time $[F(2,16)=19.47$, $p<.0002]$. There was also a significant cue lead time $\times$ 


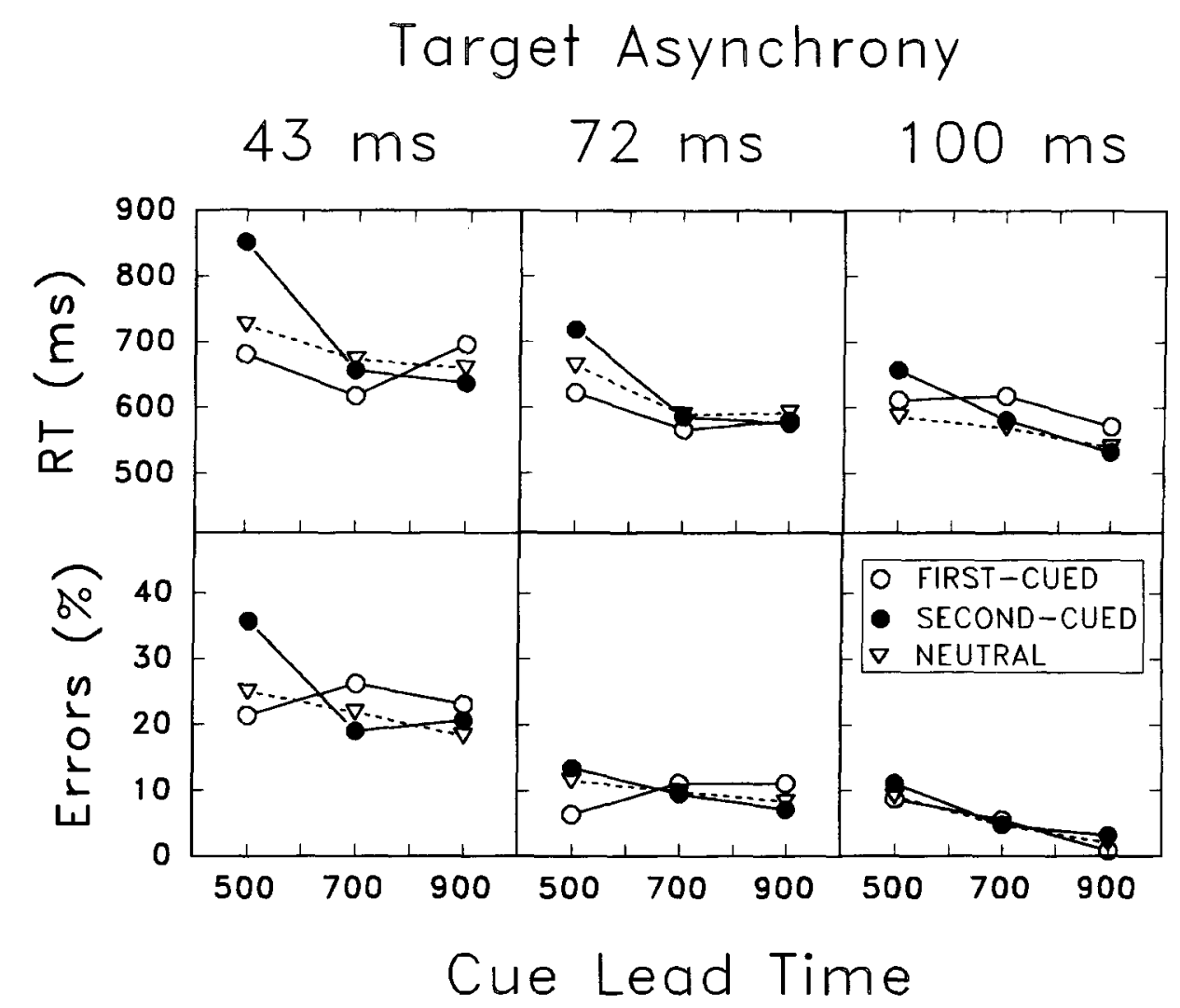

Figure 4. Mean correct response time and percent errors plotted as a function of target asynchrony, cue lead time, and cuing in Experiment 3.

cuing interaction $[F(2,16)=7.27, p<.01]$. Simple effects tests indicated that the second-cued condition differed from the neutral condition in the 500 -msec cue lead time condition $(p<.01)$ but not in the two longer cue lead time conditions (both $p s>.40$ ). Neither the target asynchrony $x$ cuing interaction nor the target asynchrony $\times$ cue lead time $\times$ cuing interaction was significant (both $F \mathrm{~s}<1$ ).

\section{Discussion}

The results of Experiment 3 yielded several important findings. First, as in Experiment 2, RTs in the secondcued condition were significantly slower than RTs in the neutral condition when cue lead time was $500 \mathrm{msec}$, but not following cue lead times of 700 or $900 \mathrm{msec}$. The results of Experiment 3 extended this finding by showing that the same RT pattern occurred regardless of target asynchrony. Together, these findings suggest that IOR is released when T1 is displayed at the uncued location. Moreover, the present findings suggest that this release mechanism operates relatively quickly, as the impairment in the second-cued condition was not altered even when the presentation of $\mathrm{T} 1$ at the uncued location preceded the presentation of $\mathrm{T} 2$ at the cued location by only $43 \mathrm{msec}$.
Second, the results of Experiment 3 showed that RTs in the first-cued condition were affected by target asynchrony. More specifically, RTs were slower in the firstcued condition than RTs in the neutral condition only when $\mathrm{T} 1$ and $\mathrm{T} 2$ were separated by $100 \mathrm{msec}$. In contrast, RTs did not differ between the two conditions at the two shorter target asynchronies (43 and $72 \mathrm{msec}$ ). This suggests that IOR may be released in a variety of stimulus situations, and provides further support for the finding that the release mechanism operates quickly. Apparently, the inhibitory processing of $\mathrm{T} 1$ at the cued location can be released by $\mathrm{T} 2$ before $\mathrm{T} 1$ reaches some critical stage of processing. Thus, at short target asynchronies, the processing of $\mathrm{T} 1$ may reflect a mixture of both inhibitory (pre-T2) and facilitatory (post-T2) processes (see General Discussion for a more detailed discussion).

The finding that IOR is released at short target asynchronies is also important because it clarifies the relationship between IOR and accuracy, at least in the TOJ task. More specifically, if latency is used as an indicator of when IOR does and does not occur in the TOJ task, then, on the one hand, accuracy should not be expected to be impaired in the first-cued condition relative to the neutral condition when target asynchrony is short (approximately $72 \mathrm{msec}$ or less) because IOR does not ap- 
pear to occur. On the other hand, IOR does occur when target asynchrony is long, and thus accuracy could, in principle, be impaired in the first-cued condition relative to the neutral condition; however, accuracy is likely at floor at these target asynchronies and therefore uninterpretable. Consequently, unlike latency measures of IOR, the present results suggest that IOR may never be shown to affect accuracy in the TOJ task. Thus, it is not surprising that previous TOJ studies (Kwak, 1992; Maylor, 1985; Posner et al., 1985) failed to show that IOR affected accuracy.

However, because evidence for IOR in the TOJ task appears to depend solely on latency, it is important to consider whether the pattern of RTs obtained in the present study might be due to some form of inhibition to respond to the cued location (Maylor, 1985; Posner et al., 1985; but see Kwak, 1992; Maylor \& Hockey, 1985) as opposed to some form of perceptual inhibition.

For instance, consider the possibility that an inhibition to respond to the cued location builds up following the cue and then begins to decay. Speeded responses associated with the first-cued condition would thus be slowed as compared with RTs associated with the neutral condition following long cue lead times, assuming the TOJ was determined before the response inhibition decayed. Importantly, such response inhibition would mask any facilitatory effects associated with the firstcued condition following long cue lead times. However, attentional facilitation may still be manifested in the second-cued condition because those responses are associated with the uncued location. Thus, response inhibition may account for the impairment observed in the first-cued condition, while facilitation may account for the impairment observed in the second-cued condition.

In addition, this response-inhibition explanation may also account for the pattern of results obtained in the first-cued condition in Experiment 3. Recall that cue lead time was always long in this experiment and that RTs in the first-cued condition were slower than RTs in the neutral condition only when the target asynchrony was $100 \mathrm{msec}$. In addition, note that the manifestation of IOR was confounded with task difficulty in this experiment; that is, performance was overall faster and more accurate as the target asynchrony increased. Hence, it is possible that response inhibition had already decayed when the target asynchrony was short because the perceptual judgment took too long to resolve. In this case, RTs associated with the first-cued condition would primarily reflect perceptual factors inherent in the task, not response inhibition. In contrast, response inhibition may not have decayed when the target asynchrony was long because the perceptual judgment was easier and therefore faster. In this case, RTs associated with the first-cued condition would be determined primarily by response inhibition and not task difficulty.

This is, in fact, the pattern of results obtained in Experiment 3. Latencies decreased across the 43-, 72-, and 100 -msec target asynchrony conditions in both the secondcued condition $(M \mathrm{~s}=715,627$, and $590 \mathrm{msec}$, respec- tively) and the neutral condition ( $M \mathrm{~s}=687,615$, and $565 \mathrm{msec}$, respectively), indicating that the task became increasingly easy as target asynchrony increased. In contrast, latencies in the first-cued condition decreased from the 43-msec target asynchrony condition $(M=665 \mathrm{msec})$ to the 72-msec condition ( $M=590 \mathrm{msec})$, but then leveled off in the $100-\mathrm{msec}$ condition $(M=600 \mathrm{msec})$. This pattern of results is consistent with the notion that response inhibition began to determine RT in the first-cued condition at some target asynchrony greater than $43 \mathrm{msec}$.

However, this response-inhibition account cannot account for the findings obtained in Experiment 1. Recall that in Experiment 1, an RT impairment was obtained in the cued condition on single-target trials when the cue lead time was $700 \mathrm{msec}$. In addition, on the basis of the results of Experiment 2, it can be inferred that RTs were also impaired in the first-cued condition on doubletarget trials in Experiment 1 when the target asynchrony was $114 \mathrm{msec}$ and the cue lead time was $700 \mathrm{msec}$. Therefore, if this response-inhibition account is correct, RTs in both the single-target cued and first-cued conditions should be equal, because they are determined by the same (response-based) inhibition. Contrary to the response-inhibition account, however, RTs were significantly slower in the first-cued condition on doubletarget trials $(M=556 \mathrm{msec})$ than they were in the cued condition on single-target trials $(M=431 \mathrm{msec})[t(12)=$ $3.56, p<.005]$, suggesting that the inhibition varied with the difficulty of the task. Hence, this response-inhibition explanation cannot account for the results obtained in the present study (see, also, Kwak, 1992; Maylor \& Hockey, 1985).

\section{GENERAL DISCUSSION}

Our primary goal in this study was to clarify the functional nature of IOR within the context of the TOJ task. The TOJ task was used because it has been taken to provide an index of the rate at which information is transmitted along visual pathways (see, e.g., Stelmach \& Herdman, 1991). As a result, the TOJ task may provide important converging evidence for the notion that IOR reflects a perceptual impairment (Abrams \& Dobkin, 1994; Posner \& Cohen, 1984). Thus, the present findings are important because they are the first to show that IOR does affect TOJs, at least in certain situations.

The TOJ task is also interesting because it differs from the more usual procedure for producing IOR in that two targets are displayed at separate locations on each trial. In contrast, initial studies of IOR (Posner \& Cohen, 1984) used a simple detection task in which a single target stimulus appeared at cued or uncued locations. Such studies identified cue lead time as the primary determinant of whether facilitation or inhibition dominated performance (Cohen, 1981; Maylor, 1985; Posner \& Cohen, 1984; Rafal et al., 1989). Consequently, cue lead time has served as the primary predictor of when IOR should and should not occur, even in tasks that differ from simple detection in potentially important ways. The present 
study calls attention to this assumption and suggests that a more complex relation between facilitatory and inhibitory processes is required when multiple targets are displayed on each trial, as in the TOJ task. In particular, whether facilitation or IOR dominates performance appears to depend on such additional factors as the temporal disparity between stimuli and the relative order in which they appear at the cued and uncued locations. How, then, should the functional relationship between facilitatory and inhibitory processes be characterized?

\section{Inhibition and Disinhibition of Return}

One possible account suggests that the release of IOR observed in the present study involves a process of active suppresion. For illustrative purposes, consider the mechanism shown in Figure 5. Following much previous research (Cohen, 1981; Maylor, 1985; Maylor \& Hockey, 1985; Posner \& Cohen, 1984; Rafal et al., 1989), this model acknowledges that facilitatory and inhibitory processes coexist at the cued location. Thus, when an exogenous cue is displayed at a particular location, both attentional (A) and inhibitory (IOR) mechanisms are activated at that location. Note, however, that this model does not attempt to explain how A or IOR operates; rather, the purpose of the model is to show that a relatively simple mechanism can account for the rather complex pattern of results obtained in the present study. Like previous conceptions of the interaction between facilitatory and inhibitory processes (Cohen, 1981; Posner \& Cohen, 1984), it is assumed that each reaches its peak at different points in time, with facilitation dominating at short cue lead times and IOR dominating at long cue lead times.

In addition, this model also includes other inhibitory connections that are activated by the cue. Unlike IOR, these other inhibitory connections do not inhibit processing at the same locations that activate them. Rather, these inhibitory connections serve to modulate the strength of IOR at other potential target locations. As such, we will refer to this type of inhibitory connection as "disinhibition of return" (DOR) to capture its proposed functional role. ${ }^{3}$

In general, the model operates in the following way: If, following the cue, a stimulus later appears at the original uncued location, then DOR becomes activated at that location, which in turn suppresses IOR at the original cued location, thus allowing any remaining facilitatory processing to influence performance at the cued location. Note that the stimulus displayed at the original uncued location also activates $\mathrm{A}$ and IOR at that location (the effects of which would be observed only if subsequent stimuli were displayed there); however, the magnitude of IOR may be smaller in this situation due to the prior activation of DOR at the original cued location. Note also that the inhibitory consequences of DOR may not be a mandatory result of stimulation at all locations, as, for instance, when stimulation occurs at fixation between the presentation of peripheral cues and targets (e.g., Posner \& Cohen, 1984). Thus, DOR may

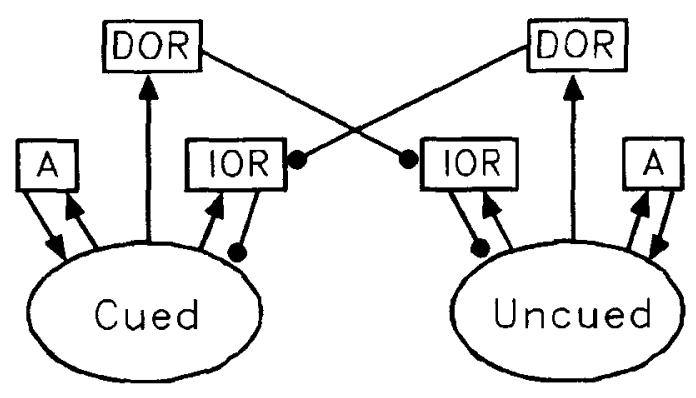

Figure 5. A model describing the functional relationship between attention, inhibition of return, and disinhibition of return at two potential target locations. Lines terminated by arrows denote facilitatory connections; lines terminated by circles denote inhibitory connections. A, attention; IOR, inhibition of return; DOR, disinhibition of return.

have functional consequences for IOR only when stimulation occurs in a potential target location; that is, DOR may depend on the experimental context in a way that IOR does not. ${ }^{4}$

According to the model, IOR may be manifest only when a stimulus displayed at the cued location reaches some critical stage of processing before DOR is activated at the uncued location. Thus, this model is consistent with findings which indicate that IOR depends on the relative temporal order of stimuli presented at the cued and uncued locations. In particular, IOR may have been suppressed by DOR when T1 was displayed at the uncued location (i.e., the second-cued condition). As a result, the processing of $\mathrm{T} 2$ at the cued location was primarily facilitated. Hence, TOJs were impaired in the second-cued condition because targets displayed at the cued location were facilitated relative to targets displayed at the uncued location.

Other evidence consistent with this aspect of the model was recently reported by Pratt and Abrams (1993) using a different (target-detection) paradigm. In their experiments, a single target was preceded on each trial by two successive cues. The interval between the first cue and the second cue was $600 \mathrm{msec}$, and the interval between the second cue and the target was $960 \mathrm{msec}$. On some trials, both cues appeared successively at the same location, in which case the target could appear either at the same location as both cues or at a location different from that of both cues. On other trials, the two cues appeared successively at different locations, in which case the target could appear either at the same location as the first cue only or at the same location as the second cue only. Thus, unlike the present study, which displayed a single cue followed by two targets, Pratt and Abrams displayed two cues followed by a single target.

Pratt and Abrams (1993) found that IOR occurred most strongly when the target appeared at the same location as both cues. More importantly, they found that IOR was eliminated when the target appeared at the same location as the first cue only. That is, IOR was eliminated when the cue immediately preceding the tar- 
get appeared at the location opposite that of the initial cue. Moreover, control studies showed that this effect was not due to the longer temporal interval separating the target and the first cue. In our view, the appearance of the second cue activates DOR at that location, which in turn inhibits IOR at the first cued location.

Interestingly, unlike the present study, Pratt and Abrams (1993) did not find facilitation at the cued location when the target appeared in the same location as the first cue only; however, the interval between the first cue and the target in their study was more than twice as long $(1,560 \mathrm{msec})$ as the longest cue lead time at which facilitation was observed in the present study. Thus, it is likely that facilitation had already dissipated before the target appeared. Finally, Pratt and Abrams also found that the effects of IOR were smaller in magnitude when the target appeared at the same location as the second cue only. As predicted, the appearance of the initial cue suppresses IOR at the second (opposite) cued location, thus making it more difficult to activate.

As in the present study, Pratt and Abrams's (1993) results indicate that IOR is constrained to occur at the most recently stimulated (or cued) location. However, Pratt and Abrams did not provide any explicit account of how IOR was released from previously stimulated locations. We have argued that IOR is actively suppressed following stimulation at other locations.

One consequence of postulating an active suppression mechanism such as DOR is that IOR may also be diminished in the first-cued condition as target asynchrony decreases. This is because the inhibitory processing of $\mathrm{T} 1$ by IOR in the first-cued condition may be suppressed by $\mathrm{T} 2$ before $\mathrm{T} 1$ reaches some critical stage of processing. In this instance, the overall processing of $\mathrm{T} 1$ at the cued location may reflect a mixture of both inhibitory (pre-T2) and facilitatory (post$\mathrm{T} 2$ ) processes. The relative proportion of inhibitory and facilitatory processing depends on target asynchrony. As shown in Experiments 2 and 3, IOR was observed only when $\mathrm{T} 1$ was displayed at the cued location $100 \mathrm{msec}$ or more before T2 was displayed at the uncued location. Presumably, IOR was observed only at these longer target asynchronies, because $\mathrm{T} 1$ was able to reach the stage of processing necessary for determining temporal order before IOR was suppressed. It should be noted, however, that target asynchrony does not provide an accurate estimate of the time required to reach this stage of processing as additional time is assumed to be required for DOR to become activated and suppress IOR.

When target asynchrony decreases below $100 \mathrm{msec}$, the processing of $\mathrm{T} 1$ at the cued location may begin to reflect a greater proportion of facilitation, assuming that the cue lead time is not beyond the point where facilitation has completely decayed, because the inhibitory processing of T1 may be suppressed earlier. This aspect of the model was also supported by the results of Experi- ment 3, which showed that RTs in the first-cued condition generally decreased relative to the neutral condition as target asynchrony decreased. That IOR can be affected by stimulation at the uncued location even in the first-cued condition is difficult to reconcile with a release mechanism that involves the passive decay of IOR; rather, the present findings seem to demand a release mechanism that actively suppresses IOR.

\section{Conclusions}

This study was conducted to clarify the functional nature of IOR. In particular, we investigated whether IOR could be shown to affect TOJs under the assumption that such an effect would reflect an impairment in perceptual processing at the cued location (Maylor, 1985). Affirmative evidence was obtained, thus strengthening Abrams and Dobkin's (1994) contention that IOR reflects, at least in part, a perceptual impairment. However, the present results also revealed a great deal more about the functional relationship between facilitatory and inhibitory processes, at least the inhibitory processes that underlie the perceptual (as opposed to oculomotor) component of IOR.

Previous studies (Posner \& Cohen, 1984) have emphasized how facilitatory and inhibitory processes interact over time to influence performance. The present study extended this line of research by showing that facilitatory and inhibitory processes can exist together in time at a single location. However, whether facilitation or inhibition dominates perceptual processing may not depend solely on how long after the cue the target appears at the cued location, especially when stimuli occur at multiple locations. Rather, interactions between facilitatory and inhibitory processes at the cued location may also be orchestrated by other inhibitory processes associated with competing locations. In particular, IOR appears to be modulated by the temporal disparity between stimuli as well as by the relative order in which they occur at cued and uncued locations. This notion of disinhibition was supported not only by the present TOJ results, but also by results obtained in a target-detection paradigm using two cues (Pratt \& Abrams, 1993). New experiments will be necessary to determine whether disinhibition also plays a role in other paradigms that have attempted, but failed, to observe IOR when two or more stimuli are displayed simultaneously (e.g., Terry, Valdes, \& Neill, 1994). For now, we conclude that IOR is a dynamic process that primarily affects the processing of temporally distinct spatial repetitions.

\section{REFERENCES}

Abrams, R. A., \& Dobkin, R. S. (1994). Inhibition of return: Effects of attentional cueing on eye movement latencies. Journal of Experimental Psychology: Human Perception \& Performance, 20, $467-$ 477.

COHEN, Y. (1981). Internal and external control of orienting. Unpublished doctoral dissertation, University of Oregon, Eugene. 
EgLy, R., RAFAl, R. D., \& HENIK, A. (1992, November). Reflexive and voluntary orienting in detection and discrimination tasks. Poster presented at the meeting of the Psychonomic Society, St. Louis.

ERIKSEN, C. W., \& YEH, Y. (1985). Allocation of attention in the visual field. Journal of Experimental Psychology: Human Perception \& Performance, 11, 583-597.

Hikosaka, O., Miyauchi, S., \& Shimojo, S. (1993). Focal visual attention produces illusory temporal order and motion sensation. $\mathrm{Vi}$ sion Research, 33, 1219-1240.

JONIDES, J. (1981). Voluntary versus automatic control over the mind's eye. In J. Long \& A. Baddeley (Eds.), Attention and performance $I X$ (pp. 187-203). Hillsdale, NJ: Erlbaum.

KWAK, H. W. (1992). Inhibitory and facilitatory components of orienting attention to locations and to features. Unpublished doctoral dissertation, Johns Hopkins University, Baltimore.

MAYLOR, E. A. (1985). Facilitory and inhibitory components of orienting in visual space. In M. I. Posner \& O. S. M. Marin (Eds.), Attention and performance XI (pp. 189-204). Hillsdale, NJ: Erlbaum.

MAYLOR, E. A., \& HOCKEY, R. (1985). Inhibitory component of externally controlled covert orienting in visual space. Journal of Experimental Psychology: Human Perception \& Performance, 11, 777-787.

PosNer, M. I., \& Boles, S. J. (1971). Components of attention. Psychological Review, 78, 391-408.

Posner, M. I., \& Cohen, Y. (1984). Components of visual orienting. In H. Bouma \& D. G. Bouwhuis (Eds.), Attention and performance $X$ (pp. 531-556). Hillsdale, NJ: Erlbaum.

Posner, M. I., Rafal, R. D., Choate, L., \& Vaughan, J. (1985). Inhibition of return: Neural basis and function. Cognitive Neuropsychology, 2, 211-228.

Pratt, J., \& ABrams, R. A. (1993, November). Inhibition of return: Effects of multiply-cued spatial locations. Poster presented at the meeting of the Psychonomic Society, Washington, DC.

Rafal, R. D., Calabresi, P. A., Brennan, C. W., \& Sciolto, T. K. (1989). Saccade preparation inhibits reorienting to recently attended locations. Journal of Experimental Psychology: Human Perception \& Performance, 15, 673-685.

Rafal, R. [D.], EgLY, R., \& Rhodes, D. (1994). Effects of inhibition of return on voluntary and visually guided saccades. Canadian Journal of Experimenal Psychology, 48, 284-300.

Stelmach, L. B., \& Herdman, C. M. (1991). Directed attention and perception of temporal order. Journal of Experimental Psychology: Human Perception \& Performance, 17, 539-550.

Terry, K. M., Valdes, L. A., \& NeIlL, W. T. (1994). Does "inhibition of return" occur in discrimination tasks? Perception \& Psychophysics, 55, 279-286.

\section{NOTES}

1. Two subjects committed $100 \%$ errors in the second-cued condition when target asynchrony was $14 \mathrm{msec}$ and cue lead time was $100 \mathrm{msec}$, and one subject committed $100 \%$ errors in the second-cued condition when target asynchrony was $14 \mathrm{msec}$ and cue lead time was $300 \mathrm{msec}$. In addition, two subjects committed $100 \%$ errors in the second-cued condition when target asynchrony was $43 \mathrm{msec}$ and cue lead time was $100 \mathrm{msec}$. These missing data were replaced by the cell mean for that condition.

2. One alternative explanation that should be considered concerns the possibility that the slower RTs observed in both the first-cued and second-cued conditions at long cue lead times reflect the same underlying deficit. In particular, the appearance of $\mathrm{T} 2$ may interfere with the execution of the intended response to $\mathrm{T} l$. If we assume that this response interference adds to the facilitatory effect of cuing, then the observed pattern of performance at short cue lead times might be predicted. That is, the difference between the first-cued condition and the neutral condition might be expected to be less than the difference between the second-cued condition and the neutral condition (see Figure 3). As facilitation diminishes, however, the difference between the first-cued and second-cued conditions should decrease, as it does. However, this response-interference hypothesis cannot account for performance at long cue lead times because it would predict not only no difference between the first-cued and second-cued conditions, but also that all three conditions (including the neutral condition) should be equally affected by the appearance of the second target. Hence, this explanation cannot account for the selective impairment observed in the first-cued and second-cued conditions relative to the neutral condition at long cue lead times.

3. Although we have depicted DOR as being directly activated by stimulation at a location, it is also theoretically possible to mediate the effects of DOR through A. At present, no conclusive evidence exists to decide this issue; hence, the model should be construed as a means of portraying some of the more general functional consequences associated with DOR rather than as a complete account of its instantiation.

4. It should be noted that Pratt and Abrams (1993) obtained evidence that IOR was eliminated when two cues occurred successively at fixation between the presentation of a peripheral cue and target, but that IOR was not eliminated when only a single cue occurred at fixation. This may suggest that DOR is only weakly activated by stimulation at a nontarget location; thus, more stimulation is required to obtain the same functional outcome as when stimulation occurs at a potential target location.

(Manuscript received March 10, 1994; revision accepted for publication June 24,1994 .) 